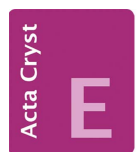

CRYSTALLOGRAPHIC COMMUNICATIONS

ISSN 2056-9890

Received 17 January 2017

Accepted 6 April 2017

Edited by M. Weil, Vienna University of Technology, Austria

Keywords: crystal structure; quasi-one-dimensional structure; mixed-metal trisulfide.

CCDC reference: 1542709

Supporting information: this article has supporting information at journals.iucr.org/e

\section{Crystal structure of the mixed-metal trisulfide $\mathrm{BaCu}_{1 / 3} \mathrm{Ta}_{2 / 3} \mathrm{~S}_{3}$}

\author{
Kejun Bu, ${ }^{\mathrm{a}}$ Jianqiao He, ${ }^{\mathrm{a}}$ Dong Wang, ${ }^{\mathrm{b}}$ Chong $\mathrm{Zheng}^{\mathrm{c} *}$ and Fuqiang Huang ${ }^{\mathrm{a} *}$
}

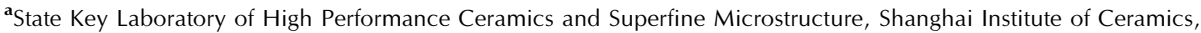 \\ Chinese Academy of Sciences, Shanghai 200050, People's Republic of China, ${ }^{\mathbf{b}}$ School of Materials Science and \\ Engineering, Shanghai University, Shangda Road, No. 99, Shanghai 200444, People's Republic of China, and \\ 'Department of Chemistry and Biochemistry, Northern Illinois University, USA. *Correspondence e-mail: \\ czheng@niu.edu, huangfq@mail.sic.ac.cn
}

The mixed-metal title compound, $\mathrm{BaCu}_{1 / 3} \mathrm{Ta}_{2 / 3} \mathrm{~S}_{3}$ [barium copper(II) tanta$\operatorname{lum}(\mathrm{V})$ trisulfide], was prepared through solid-state reactions. The crystal structure adopts the $\mathrm{BaTaS}_{3}$ structure type and consists of face-sharing $\left[M \mathrm{~S}_{6}\right](M$ $=\mathrm{Ta}, \mathrm{Cu}$ ) octahedra (point-group symmetry $\overline{3} \mathrm{~m}$.) that are condensed into infinite chains along [001]. Adjacent chains are linked through the barium cations (site symmetry $\overline{6} m 2$ ), which exhibit a coordination number of twelve. The $M$ site is occupied by $2 / 3$ of $\mathrm{Ta}^{\mathrm{V}}$ and $1 / 3$ of $\mathrm{Cu}^{\mathrm{II}}$, whereby the average $M-\mathrm{S}$ distances are slightly longer than those of ordered $\mathrm{BaTaS}_{3}$. The classical charge balance of the title compound can be represented by $\left[\mathrm{Ba}^{2+}\right]\left[(\mathrm{Ta} / \mathrm{Cu})^{4+}\right]\left[\mathrm{S}^{2-}\right]_{3}$.

\section{Chemical context}

Barium vanadium trisulfide, $\mathrm{BaVS}_{3}$ (Takano et al., 1977), with which $\mathrm{BaTaS}_{3}$ (Gardner et al., 1969) crystallizes isotypically in space group $P 6_{3} / m m c$, has a chain structure. The observed conductivity was attributed to the formation of conduction bands via vanadium...vanadium $d$-orbital overlap. It shows three phase transitions and exhibits a number of intriguing physical properties (Nakamura et al., 1994). While both $\mathrm{BaVS}_{3}$ and $\mathrm{BaTaS}_{3}$ are composed of the same type of linear chains, $\mathrm{BaTaS}_{3}$ shows metallic conductivity and a Curie-Weiss behaviour of the magnetic susceptibility (Gardner et al., 1969). To explore the physical properties of $\mathrm{BaTaS}_{3}$ and related

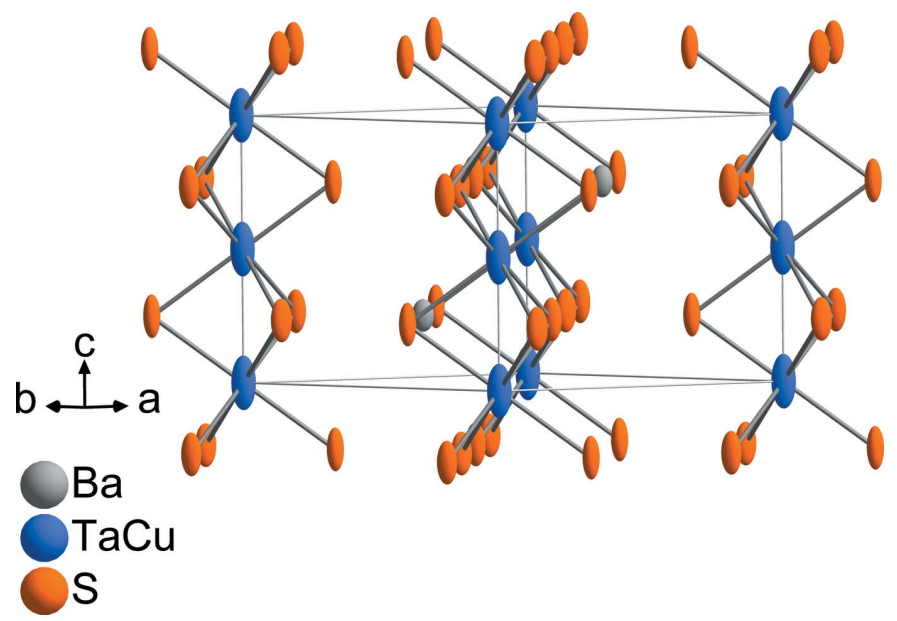

Figure 1

Face-sharing of $M \mathrm{~S}_{6}(M=\mathrm{Cu}, \mathrm{Ta})$ octahedra in the structure of $\mathrm{BaCu}_{1 / 3^{-}}$ $\mathrm{Ta}_{2 / 3} \mathrm{~S}_{3}$. Displacement ellipsoids are drawn at the $50 \%$ probability level. 


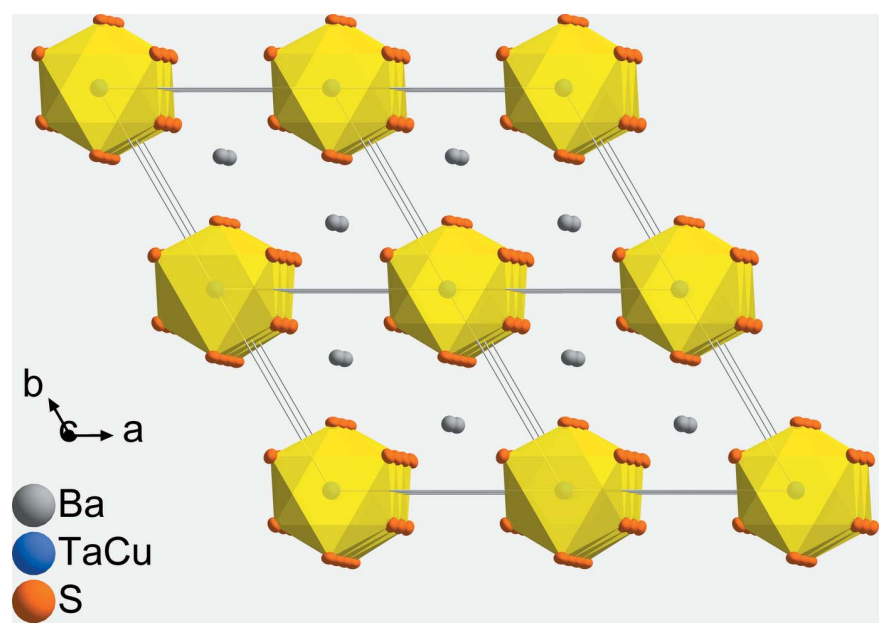

Figure 2

The crystal structure of $\mathrm{BaCu}_{1 / 3} \mathrm{Ta}_{2 / 3} \mathrm{~S}_{3}$, viewed down [001].

compounds, we have introduced copper and studied mixedmetal phases $\mathrm{Ba}(\mathrm{Ta} / \mathrm{Cu}) \mathrm{S}_{3}$. Here we report on the synthesis and structural characterization of the mixed-metal trisulfide with composition $\mathrm{BaCu}_{1 / 3} \mathrm{Ta}_{2 / 3} \mathrm{~S}_{3}$.

\section{Structural commentary}

$\mathrm{BaCu}_{1 / 3} \mathrm{Ta}_{2 / 3} \mathrm{~S}_{3}$ adopts the $\mathrm{BaTaS}_{3}$ structure type in space group $P 6_{3} / m m c$. A detailed description of this structure type has been given previously (Gardner et al., 1969). The asymmetric unit of $\mathrm{BaCu}_{1 / 3} \mathrm{Ta}_{2 / 3} \mathrm{~S}_{3}$ contains one Ba site (Wyckoff position $2 c)$, one mixed-occupied $(\mathrm{Cu} / \mathrm{Ta})$ site $(2 a)$ and one $\mathrm{S}$ site $(6 h)$. The structure contains face-sharing octahedral $\left[M \mathrm{~S}_{6}\right]$ $(M=\mathrm{Cu}, \mathrm{Ta})$ units, which construct infinite chains along [001] (Fig. 1). In the crystal structure, these chains are linked through $\mathrm{Ba}$ cations (coordination number 12) to adjacent chains (Fig. 2).

The $M$ site is occupationally disordered and contains $1 / 3 \mathrm{Cu}$ and 2/3 Ta. It is surrounded by six $\mathrm{S}$ atoms with an $M-\mathrm{S}$ bond length of 2.475 (4) $\AA$, which is slightly longer than that of ordered $\mathrm{BaTaS}_{3}$ (2.461 $\AA$; Gardner et al., 1969). This trend is in agreement with the different ionic radii of Ta $\left(0.64 \AA\right.$ for $\mathrm{Ta}^{\mathrm{V}}$ with coordination number of six) and $\mathrm{Cu}^{\mathrm{II}}(0.73 \AA)$ using the data provided by Shannon (1976).

The $(\mathrm{Cu}, \mathrm{Ta}) \cdots(\mathrm{Cu}, \mathrm{Ta})$ distance within a chain is 2.9159 (3) $\AA$, which is much shorter than the interchain $(\mathrm{Cu}, \mathrm{Ta}) \cdots(\mathrm{Cu}, \mathrm{Ta})$ distance of $6.8437(18) \AA$. The $\mathrm{Ba}-\mathrm{S}$ interactions between adjacent metal sulfide chains are reflected by one shorter [3.422 (6) $\AA$ ] and one longer distance [3.523 (3) ̊], in good agreement with those found in other barium tantalum sulfides (Onoda \& Saeki, 1989).

The classical charge balance of the title compound can be represented by the formula $\left[\mathrm{Ba}^{2+}\right]\left[(\mathrm{Ta} / \mathrm{Cu})^{4+}\right]\left[\mathrm{S}^{2-}\right]_{3}$.

\section{Synthesis and crystallization}

The title compound was prepared using solid-state reactions between the elements $\mathrm{Cu}, \mathrm{Ta}, \mathrm{S}$ and BaS. Ta powder $(99.999 \%$,
Table 1

Experimental details.
Crystal data

Chemical formula

$M_{\text {r }}$

Crystal system, space group

Temperature (K)

$a, c(\AA)$

$V\left(\AA^{3}\right)$

Z

Radiation type

$\mu\left(\mathrm{mm}^{-1}\right)$

Crystal size (mm)

Data collection

Diffractometer

Absorption correction

$T_{\min }, T_{\max }$

No. of measured, independent and observed $[I>2 \sigma(I)]$ reflections

$R_{\text {int }}$

$(\sin \theta / \lambda)_{\max }\left(\AA^{-1}\right)$

Refinement

$R\left[F^{2}>2 \sigma\left(F^{2}\right)\right], w R\left(F^{2}\right), S$

No. of reflections

No. of parameters

No. of restraints

$\Delta \rho_{\max }, \Delta \rho_{\min }\left(\mathrm{e} \AA^{-3}\right)$

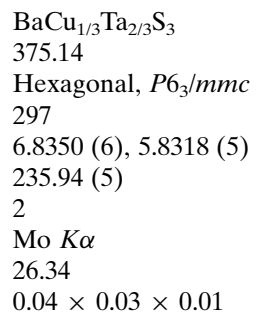

Bruker D8 QUEST

Multi-scan ( $S A D A B S$; Bruker, 2015)

$0.44,0.86$

$4312,125,106$

0.042

0.645

$0.043,0.113,1.25$

125

11

$1.50,-1.50$
Computer programs: APEX3 and SAINT (Bruker, 2015), SHELXT (Sheldrick, 2015a), SHELXL2014/7 (Sheldrick, 2015b), DIAMOND (Brandenburg, 2007) and publCIF (Westrip, 2010).

Alfa Aesar Puratronic), Cu powder (99.999\%, Alfa Aesar Puratronic), S powder (99.999\%, Alfa Aesar Puratronic), and BaS powder (99.999\%, Alfa Aesar Puratronic) were mixed in a fused-silica tube in an Ta:Cu:S:BaS molar ratio of 0.67:0.33:2:1. The tube was evacuated to $0.1 \mathrm{~Pa}$, sealed and heated gradually $\left(60 \mathrm{~K} \mathrm{~h}^{-1}\right)$ to $973 \mathrm{~K}$, where it was kept for $2 \mathrm{~d}$. The tube was then cooled to $673 \mathrm{~K}$ at a rate of $3 \mathrm{~K} \mathrm{~h}^{-1}$ and then quenched to room temperature. The crystals are stable in air and alcohol.

Scanning electron microscopy (SEM) images of selected crystals were taken on a Hitachi S-4800 microscope equipped with an electron microprobe analyzer for a semiquantitative elemental analysis in the energy dispersive X-ray spectroscopy (EDX) mode. The presence of both copper and tantalum was confirmed (Fig. 3).
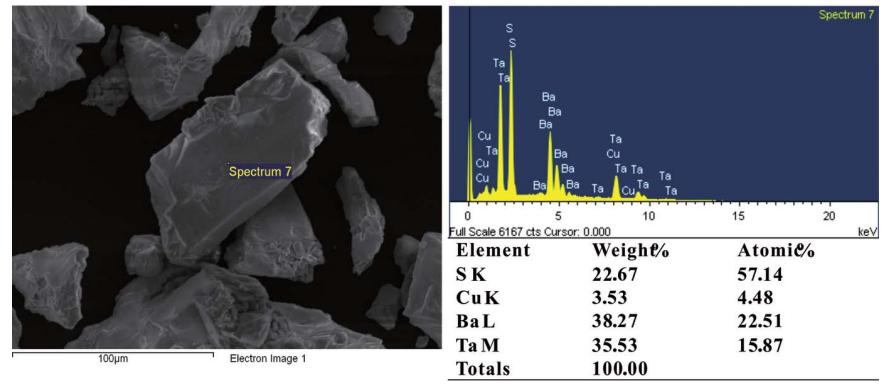

Figure 3

SEM image and EDX spectrum of $\mathrm{BaCu}_{1 / 3} \mathrm{Ta}_{2 / 3} \mathrm{~S}_{3}$. 


\section{Refinement}

Crystal data, data collection and structure refinement details are summarized in Table 1 . The refinement of the model with occupational disorder on the $M$ site resulted in a significant decrease of the reliability factors in comparison with a fully occupied Ta site $(R 1=0.73, w R=0.197)$. No evidence, $e . g$. in the form of superstructure reflections, was found for an ordering of this site and thus a statistically disordered model was considered. In the final model, atoms of the disordered site were restrained to have the same displacement parameters, with a fixed $\mathrm{Cu}$ :Ta ratio of 1/3:2/3 required for charge neutrality and in good agreement with the EDX measurement. The remaining maximum and minimum electron densities are located $1.06 \AA$ from the $(\mathrm{Cu}, \mathrm{Ta}) 1$ site and $1.96 \AA$ from the $\mathrm{S} 1$, respectively.

\section{Funding information}

Funding for this research was provided by: State Key Laboratory of High Performance Ceramics and Superfine Microstructure, Shanghai Institute of Ceramics, Chinese Academy of Sciences, Shanghai 200050, People's Republic of
China. (award No. XDB04040200); CAS Center for Excellence in Superconducting Electronics, National key research and development program (award No. 2016YFB0901600); NSF of China (award Nos. 11404358, 51402341); Science and Technology Commission of Shanghai (award Nos. 13JC1405700, 14520722000).

\section{References}

Brandenburg, K. (2007). DIAMOND. Crystal Impact GbR, Bonn, Germany.

Bruker (2015). APEX3, SAINT and SADABS. Bruker AXS Inc., Madison, Wisconsin, USA.

Gardner, R. A., Vlasse, M. \& Wold, A. (1969). Inorg. Chem. 8, 2784 2787.

Nakamura, M., Sekiyama, A., Namatame, H., Fujimori, A., Yoshihara, H., Ohtani, T., Misu, A. \& Takano, M. (1994). Phys. Rev. B, 49, 16191-16201.

Onoda, M. \& Saeki, M. (1989). Mater. Res. Bull. 24, 625-631.

Shannon, R. D. (1976). Acta Cryst. A32, 751-767.

Sheldrick, G. M. (2015a). Acta Cryst. A71, 3-8.

Sheldrick, G. M. (2015b). Acta Cryst. C71, 3-8.

Takano, M., Kosugi, H., Nakanishi, N., Shimada, M., Wada, T. \& Koizumi, M. (1977). J. Phys. Soc. Jpn, 43, 1101-1102.

Westrip, S. P. (2010). J. Appl. Cryst. 43, 920-925. 


\section{supporting information}

Acta Cryst. (2017). E73, 713-715 [https://doi.org/10.1107/S2056989017005266]

\section{Crystal structure of the mixed-metal trisulfide $\mathrm{BaCu}_{1 / 3} \mathrm{Ta}_{2 / 3} \mathrm{~S}_{3}$}

\section{Kejun Bu, Jianqiao He, Dong Wang, Chong Zheng and Fuqiang Huang}

Computing details

Data collection: APEX3 (Bruker, 2015); cell refinement: SAINT (Bruker, 2015); data reduction: SAINT (Bruker, 2015); program(s) used to solve structure: SHELXT (Sheldrick, 2015a); program(s) used to refine structure: SHELXL2014/7 (Sheldrick, 2015b); molecular graphics: DIAMOND (Brandenburg, 2007); software used to prepare material for publication: publCIF (Westrip, 2010).

Barium copper(II) tantalum(V) trisulfide

Crystal data

$\mathrm{BaCu}_{0.33} \mathrm{Ta}_{0.67} \mathrm{~S}_{3}$

$D_{\mathrm{x}}=5.280 \mathrm{Mg} \mathrm{m}^{-3}$

$M_{r}=375.14$

Hexagonal, $P 6_{3} / m m c$

$a=6.8350(6) \AA$

$c=5.8318(5) \AA$

$V=235.94(5) \AA^{3}$

Mo $K \alpha$ radiation, $\lambda=0.71073 \AA$

$Z=2$

$F(000)=325$

Cell parameters from 1738 reflections

$\theta=3.4-25.9^{\circ}$

$\mu=26.34 \mathrm{~mm}^{-1}$

$T=297 \mathrm{~K}$

Plate, black

$0.04 \times 0.03 \times 0.01 \mathrm{~mm}$

Data collection

Bruker D8 QUEST

125 independent reflections

diffractometer

106 reflections with $I>2 \sigma(I)$

Detector resolution: 10.4167 pixels $\mathrm{mm}^{-1}$

$R_{\text {int }}=0.042$

phi and $\omega$ scans

Absorption correction: multi-scan

$\theta_{\max }=27.3^{\circ}, \theta_{\min }=3.4^{\circ}$

(SADABS; Bruker, 2015)

$T_{\text {min }}=0.44, T_{\max }=0.86$

$h=-8 \rightarrow 8$

$k=-8 \rightarrow 8$

$l=-7 \rightarrow 6$

4312 measured reflections

\section{Refinement}

Refinement on $F^{2}$

Least-squares matrix: full

$R\left[F^{2}>2 \sigma\left(F^{2}\right)\right]=0.043$

$w R\left(F^{2}\right)=0.113$

$S=1.25$

125 reflections

11 parameters

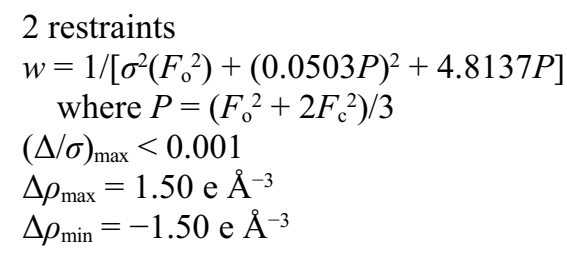




\section{Special details}

Geometry. All esds (except the esd in the dihedral angle between two 1.s. planes) are estimated using the full covariance matrix. The cell esds are taken into account individually in the estimation of esds in distances, angles and torsion angles; correlations between esds in cell parameters are only used when they are defined by crystal symmetry. An approximate (isotropic) treatment of cell esds is used for estimating esds involving l.s. planes.

Fractional atomic coordinates and isotropic or equivalent isotropic displacement parameters $\left(\hat{A}^{2}\right)$

\begin{tabular}{llllll}
\hline & $x$ & $y$ & $z$ & $U_{\text {iso }} * U_{\text {eq }}$ & Occ. $(<1)$ \\
\hline $\mathrm{Ba}$ & 0.6667 & 0.3333 & 0.75 & $0.0341(7)$ & \\
$\mathrm{Ta}$ & 0 & 0 & 0.5 & $0.0707(12)$ & $0.6666(8)$ \\
$\mathrm{Cu}$ & 0 & 0 & 0.5 & $0.0707(12)$ & $0.3334(18)$ \\
$\mathrm{S}$ & $0.1689(4)$ & $0.3378(8)$ & 0.75 & $0.0449(15)$ & \\
\hline
\end{tabular}

Atomic displacement parameters $\left(\AA^{2}\right)$

\begin{tabular}{lllllll}
\hline & $U^{11}$ & $U^{22}$ & $U^{33}$ & $U^{12}$ & $U^{13}$ & $U^{23}$ \\
\hline $\mathrm{Ba}$ & $0.0246(7)$ & $0.0246(7)$ & $0.0529(13)$ & $0.0123(4)$ & 0 & 0 \\
$\mathrm{Ta}$ & $0.0306(8)$ & $0.0306(8)$ & $0.151(3)$ & $0.0153(4)$ & 0 & 0 \\
$\mathrm{Cu}$ & $0.0306(8)$ & $0.0306(8)$ & $0.151(3)$ & $0.0153(4)$ & 0 & 0 \\
$\mathrm{~S}$ & $0.0217(18)$ & $0.015(2)$ & $0.096(4)$ & $0.0074(10)$ & 0 & 0 \\
\hline
\end{tabular}

Geometric parameters $\left(\AA,{ }^{o}\right)$

\begin{tabular}{|c|c|c|c|}
\hline $\mathrm{Ba}-\mathrm{S}^{\mathrm{i}}$ & $3.4176(3)$ & $\mathrm{Ta}-\mathrm{S}$ & $2.475(4)$ \\
\hline $\mathrm{Ba}-\mathrm{S}^{\mathrm{ii}}$ & $3.4176(3)$ & $\mathrm{Ta}-\mathrm{S}^{\mathrm{xiii}}$ & $2.475(4)$ \\
\hline $\mathrm{Ba}-\mathrm{S}^{\mathrm{iii}}$ & $3.4176(3)$ & $\mathrm{Ta}-\mathrm{S}^{\mathrm{vii}}$ & $2.475(4)$ \\
\hline $\mathrm{Ba}-\mathrm{S}^{\mathrm{iv}}$ & $3.4176(3)$ & $\mathrm{Ta}-\mathrm{S}^{\mathrm{xiv}}$ & $2.475(4)$ \\
\hline $\mathrm{Ba}-\mathrm{S}$ & $3.4176(3)$ & $\mathrm{Ta}-\mathrm{Ta}^{\mathrm{xv}}$ & $2.9159(3)$ \\
\hline $\mathrm{Ba}-\mathrm{S}^{\mathrm{v}}$ & $3.4176(3)$ & $\mathrm{Ta}-\mathrm{Cu}^{\mathrm{xvi}}$ & $2.9159(3)$ \\
\hline $\mathrm{Ba}-\mathrm{S}^{\mathrm{vi}}$ & $3.506(3)$ & $\mathrm{Ta}-\mathrm{Cu}^{\mathrm{xv}}$ & $2.9159(3)$ \\
\hline $\mathrm{Ba}-\mathrm{S}^{\mathrm{vii}}$ & $3.506(3)$ & $\mathrm{Ta}-\mathrm{Ta}^{\mathrm{xvi}}$ & $2.9159(3)$ \\
\hline $\mathrm{Ba}-\mathrm{S}^{\text {viii }}$ & $3.506(3)$ & $\mathrm{S}-\mathrm{Cu}^{\mathrm{xv}}$ & $2.475(4)$ \\
\hline $\mathrm{Ba}-\mathrm{S}^{\mathrm{ix}}$ & $3.506(3)$ & $\mathrm{S}-\mathrm{Ta}^{\mathrm{xv}}$ & $2.475(4)$ \\
\hline $\mathrm{Ba}-\mathrm{S}^{\mathrm{x}}$ & $3.506(3)$ & $\mathrm{S}-\mathrm{Ba}^{\mathrm{xvii}}$ & $3.4176(3)$ \\
\hline $\mathrm{Ba}-\mathrm{S}^{\mathrm{xi}}$ & $3.506(3)$ & $\mathrm{S}-\mathrm{Ba}^{\mathrm{vi}}$ & $3.506(3)$ \\
\hline $\mathrm{Ta}-\mathrm{S}^{\mathrm{xii}}$ & $2.475(4)$ & $\mathrm{S}-\mathrm{Ba}^{\mathrm{ix}}$ & $3.506(3)$ \\
\hline $\mathrm{Ta}-\mathrm{S}^{\mathrm{iii}}$ & $2.475(4)$ & & \\
\hline $\mathrm{S}^{\mathrm{i}}-\mathrm{Ba}-\mathrm{S}^{\mathrm{ii}}$ & $60.89(16)$ & 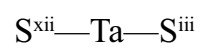 & 180.0 \\
\hline $\mathrm{S}^{\mathrm{i}}-\mathrm{Ba}-\mathrm{S}^{\mathrm{iii}}$ & $120.0010(10)$ & $\mathrm{S}^{\mathrm{xii}}-\mathrm{Ta}-\mathrm{S}$ & $91.18(10)$ \\
\hline $\mathrm{S}^{\mathrm{ii}}-\mathrm{Ba}-\mathrm{S}^{\mathrm{iii}}$ & $59.11(17)$ & $\mathrm{S}^{\mathrm{iii}}-\mathrm{Ta}-\mathrm{S}$ & $88.82(10)$ \\
\hline 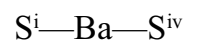 & $59.11(17)$ & $\mathrm{S}^{\mathrm{xii}}-\mathrm{Ta}-\mathrm{S}^{\mathrm{xiii}}$ & $88.82(10)$ \\
\hline $\mathrm{S}^{\mathrm{ii}}-\mathrm{Ba}-\mathrm{S}^{\mathrm{iv}}$ & $120.0010(10)$ & $\mathrm{S}^{\mathrm{iii}}-\mathrm{Ta}-\mathrm{S}^{\mathrm{xiii}}$ & $91.18(10)$ \\
\hline $\mathrm{S}^{\mathrm{iii}}-\mathrm{Ba}-\mathrm{S}^{\mathrm{iv}}$ & $179.11(16)$ & $\mathrm{S}-\mathrm{Ta}-\mathrm{S}^{\mathrm{xiii}}$ & 180.0 \\
\hline $\mathrm{S}-\mathrm{i}-\mathrm{Ba}-\mathrm{S}$ & $179.11(17)$ & $\mathrm{S}^{\mathrm{xii}}-\mathrm{Ta}-\mathrm{S}^{\mathrm{vii}}$ & $88.82(10)$ \\
\hline $\mathrm{S}^{\mathrm{ii}}-\mathrm{Ba}-\mathrm{S}$ & $120.0000(10)$ & $\mathrm{S}^{\mathrm{iii}}-\mathrm{Ta}-\mathrm{S}^{\mathrm{vii}}$ & $91.18(10)$ \\
\hline $\mathrm{S}^{\mathrm{iii}-\mathrm{Ba}-\mathrm{S}}$ & $60.89(17)$ & $\mathrm{S}-\mathrm{Ta}-\mathrm{S}^{\mathrm{vii}}$ & $91.18(10)$ \\
\hline
\end{tabular}




\begin{tabular}{|c|c|}
\hline $\mathrm{S}^{\mathrm{iv}}-\mathrm{Ba}-\mathrm{S}$ & $119.9990(10)$ \\
\hline $\mathrm{S}-\mathrm{Ba}-\mathrm{S}^{\mathrm{v}}$ & $120.0000(10)$ \\
\hline $\mathrm{S}^{\mathrm{ii}}-\mathrm{Ba}-\mathrm{S}^{\mathrm{v}}$ & $179.11(17)$ \\
\hline $\mathrm{S}^{\mathrm{iii}}-\mathrm{Ba}-\mathrm{S}^{\mathrm{v}}$ & $119.9990(10)$ \\
\hline $\mathrm{S}^{\mathrm{iv}}-\mathrm{Ba}-\mathrm{S}^{\mathrm{v}}$ & $60.89(17)$ \\
\hline $\mathrm{S}-\mathrm{Ba}-\mathrm{S}^{\mathrm{v}}$ & $59.11(17)$ \\
\hline $\mathrm{S}^{\mathrm{i}}-\mathrm{Ba}-\mathrm{S}^{\mathrm{vi}}$ & $89.75(5)$ \\
\hline $\mathrm{S}^{\mathrm{ii}}-\mathrm{Ba}-\mathrm{S}^{\mathrm{vi}}$ & $118.88(3)$ \\
\hline $\mathrm{S}^{\mathrm{iii}}-\mathrm{Ba}-\mathrm{S}^{\mathrm{vi}}$ & $118.88(3)$ \\
\hline $\mathrm{S}^{\mathrm{iv}}-\mathrm{Ba}-\mathrm{S}^{\mathrm{vi}}$ & $61.40(8)$ \\
\hline $\mathrm{S}-\mathrm{Ba}-\mathrm{S}^{\mathrm{vi}}$ & $89.75(5)$ \\
\hline $\mathrm{S}^{\mathrm{v}}-\mathrm{Ba}-\mathrm{S}^{\mathrm{vi}}$ & $61.40(8)$ \\
\hline $\mathrm{S}^{\mathrm{i}}-\mathrm{Ba}-\mathrm{S}^{\mathrm{vii}}$ & $118.88(3)$ \\
\hline $\mathrm{S}^{\mathrm{ii}}-\mathrm{Ba}-\mathrm{S}^{\mathrm{vii}}$ & $89.75(5)$ \\
\hline $\mathrm{S}^{\mathrm{iii}}-\mathrm{Ba}-\mathrm{S}^{\mathrm{vii}}$ & $61.40(8)$ \\
\hline $\mathrm{S}^{\mathrm{iv}}-\mathrm{Ba}-\mathrm{S}^{\mathrm{vii}}$ & $118.88(3)$ \\
\hline $\mathrm{S}-\mathrm{Ba}-\mathrm{S}^{\mathrm{vii}}$ & $61.40(8)$ \\
\hline $\mathrm{S}^{\mathrm{v}}-\mathrm{Ba}-\mathrm{S}^{\mathrm{vii}}$ & $89.75(5)$ \\
\hline $\mathrm{S}^{\mathrm{vi}}-\mathrm{Ba}-\mathrm{S}^{\mathrm{vii}}$ & $147.76(6)$ \\
\hline $\mathrm{S}^{\mathrm{i}-\mathrm{Ba}-\mathrm{S}^{\text {viii }}}$ & $118.88(3)$ \\
\hline $\mathrm{S}^{\mathrm{ii}}-\mathrm{Ba}-\mathrm{S}^{\mathrm{viii}}$ & $89.75(5)$ \\
\hline $\mathrm{S}^{\mathrm{iii}}-\mathrm{Ba}-\mathrm{S}^{\mathrm{viii}}$ & $61.40(8)$ \\
\hline $\mathrm{S}^{\mathrm{iv}}-\mathrm{Ba}-\mathrm{S}^{\text {viii }}$ & $118.88(3)$ \\
\hline $\mathrm{S}-\mathrm{Ba}-\mathrm{S}^{\text {viii }}$ & $61.40(8)$ \\
\hline $\mathrm{S}^{\mathrm{v}}-\mathrm{Ba}-\mathrm{S}^{\mathrm{viii}}$ & $89.75(5)$ \\
\hline $\mathrm{S}^{\mathrm{vi}}-\mathrm{Ba}-\mathrm{S}^{\mathrm{viii}}$ & $57.48(11)$ \\
\hline $\mathrm{S}^{\mathrm{vii}}-\mathrm{Ba}-\mathrm{S}^{\mathrm{vii}}$ & $112.55(14)$ \\
\hline $\mathrm{S}-\mathrm{Ba}-\mathrm{S}^{\mathrm{ix}}$ & $89.75(5)$ \\
\hline $\mathrm{S}^{\mathrm{ii}}-\mathrm{Ba}-\mathrm{S}^{\mathrm{ix}}$ & $118.88(3)$ \\
\hline 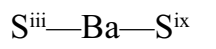 & $118.88(3)$ \\
\hline $\mathrm{S}^{\mathrm{iv}}-\mathrm{Ba}-\mathrm{S}^{\mathrm{ix}}$ & $61.40(8)$ \\
\hline $\mathrm{S}-\mathrm{Ba}-\mathrm{S}^{\mathrm{ix}}$ & $89.75(5)$ \\
\hline$S^{v}-B a-S^{i x}$ & $61.40(8)$ \\
\hline $\mathrm{S}^{\mathrm{vi}}-\mathrm{Ba}-\mathrm{S}^{\mathrm{ix}}$ & $112.55(14)$ \\
\hline $\mathrm{S}^{\mathrm{vii}}-\mathrm{Ba}-\mathrm{S}^{\mathrm{ix}}$ & $57.48(11)$ \\
\hline 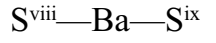 & $147.76(6)$ \\
\hline 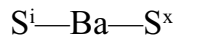 & $61.40(8)$ \\
\hline $\mathrm{S}^{\mathrm{ii}}-\mathrm{Ba}-\mathrm{S}^{\mathrm{x}}$ & $61.40(8)$ \\
\hline $\mathrm{S}^{\mathrm{iii}}-\mathrm{Ba}-\mathrm{S}^{\mathrm{x}}$ & $89.75(5)$ \\
\hline $\mathrm{S}^{\mathrm{iv}}-\mathrm{Ba}-\mathrm{S}^{\mathrm{x}}$ & $89.75(5)$ \\
\hline $\mathrm{S}-\mathrm{Ba}-\mathrm{S}^{\mathrm{x}}$ & $118.88(3)$ \\
\hline$S^{v}-B a-S^{x}$ & $118.88(3)$ \\
\hline $\mathrm{S}^{\mathrm{vi}}-\mathrm{Ba}-\mathrm{S}^{\mathrm{x}}$ & $57.48(11)$ \\
\hline $\mathrm{S}^{\mathrm{vii}}-\mathrm{Ba}-\mathrm{S}^{\mathrm{x}}$ & $147.76(6)$ \\
\hline $\mathrm{S}^{\mathrm{viii}-\mathrm{Ba}-\mathrm{S}^{\mathrm{x}}}$ & $57.48(11)$ \\
\hline $\mathrm{S}^{\mathrm{ix}}-\mathrm{Ba}-\mathrm{S}^{\mathrm{x}}$ & $147.76(6)$ \\
\hline $\mathrm{S}^{\mathrm{i}}-\mathrm{Ba}-\mathrm{S}^{\mathrm{xi}}$ & $61.40(8)$ \\
\hline $\mathrm{Sii}_{\mathrm{ii}} \quad \mathrm{B}_{\mathrm{a}} \quad \mathrm{Xi}$ & \\
\hline
\end{tabular}

\begin{tabular}{|c|c|}
\hline $\mathrm{S}^{\mathrm{xiii}}-\mathrm{Ta}-\mathrm{S}^{\mathrm{vii}}$ & $88.82(10)$ \\
\hline $\mathrm{S}^{\mathrm{xii}}-\mathrm{Ta}-\mathrm{S}^{\mathrm{xiv}}$ & $91.18(10)$ \\
\hline $\mathrm{S}^{\mathrm{iii}}-\mathrm{Ta}-\mathrm{S}^{\mathrm{xiv}}$ & $88.82(10)$ \\
\hline $\mathrm{S}-\mathrm{Ta}-\mathrm{S}^{\mathrm{xiv}}$ & $88.82(10)$ \\
\hline $\mathrm{S}^{\mathrm{xii}}-\mathrm{Ta}-\mathrm{S}^{\mathrm{xiv}}$ & $91.18(10)$ \\
\hline $\mathrm{S}^{\mathrm{vii}}-\mathrm{Ta}-\mathrm{S}^{\mathrm{xiv}}$ & 180.0 \\
\hline $\mathrm{S}^{\mathrm{xii}}-\mathrm{Ta}-\mathrm{Ta}^{\mathrm{xv}}$ & $126.10(7)$ \\
\hline $\mathrm{S}^{\mathrm{iii}}-\mathrm{Ta}-\mathrm{Ta}^{\mathrm{xv}}$ & $53.90(7)$ \\
\hline $\mathrm{S}-\mathrm{Ta}-\mathrm{Ta}^{\mathrm{xv}}$ & $53.90(7)$ \\
\hline $\mathrm{S}^{\mathrm{xii}}-\mathrm{Ta}-\mathrm{Ta}^{\mathrm{xv}}$ & $126.10(7)$ \\
\hline $\mathrm{S}^{\mathrm{vii}}-\mathrm{Ta}-\mathrm{Ta}^{\mathrm{xv}}$ & $126.10(7)$ \\
\hline $\mathrm{S}^{\mathrm{xiv}}-\mathrm{Ta}-\mathrm{Ta}^{\mathrm{xv}}$ & $53.90(7)$ \\
\hline $\mathrm{S}^{\mathrm{xii}}-\mathrm{Ta}-\mathrm{Cu}^{\mathrm{xvi}}$ & $53.90(7)$ \\
\hline $\mathrm{S}^{\mathrm{iii}}-\mathrm{Ta}-\mathrm{Cu}^{\mathrm{xvi}}$ & $126.10(7)$ \\
\hline $\mathrm{S}-\mathrm{Ta}-\mathrm{Cu}^{\mathrm{xvi}}$ & $126.10(7)$ \\
\hline $\mathrm{S}^{\mathrm{xiii}}-\mathrm{Ta}-\mathrm{Cu}^{\mathrm{xvi}}$ & $53.90(7)$ \\
\hline $\mathrm{S}^{\mathrm{vii}}-\mathrm{Ta}-\mathrm{Cu}^{\mathrm{xvi}}$ & $53.90(7)$ \\
\hline $\mathrm{S}^{\mathrm{xiv}}-\mathrm{Ta}-\mathrm{Cu}^{\mathrm{xvi}}$ & $126.10(7)$ \\
\hline $\mathrm{Ta}^{\mathrm{xv}}-\mathrm{Ta}-\mathrm{Cu}^{\mathrm{xvi}}$ & 180.0 \\
\hline $\mathrm{S}^{\mathrm{xii}}-\mathrm{Ta}-\mathrm{Cu}^{\mathrm{xv}}$ & $126.10(7)$ \\
\hline $\mathrm{S}^{\mathrm{iii}}-\mathrm{Ta}-\mathrm{Cu}^{\mathrm{xv}}$ & $53.90(7)$ \\
\hline $\mathrm{S}-\mathrm{Ta}-\mathrm{Cu}^{\mathrm{xv}}$ & $53.90(7)$ \\
\hline $\mathrm{S}^{\mathrm{xii}}-\mathrm{Ta}-\mathrm{Cu}^{\mathrm{xv}}$ & $126.10(7)$ \\
\hline $\mathrm{S}^{\mathrm{vii}}-\mathrm{Ta}-\mathrm{Cu}^{\mathrm{xv}}$ & $126.10(7)$ \\
\hline $\mathrm{S}^{\mathrm{xiv}}-\mathrm{Ta}-\mathrm{Cu}^{\mathrm{xv}}$ & $53.90(7)$ \\
\hline $\mathrm{Ta}^{\mathrm{xv}}-\mathrm{Ta}-\mathrm{Cu}^{\mathrm{xv}}$ & 0 \\
\hline $\mathrm{Cu}^{\mathrm{xvi}}-\mathrm{Ta}-\mathrm{Cu}^{\mathrm{xv}}$ & 180.0 \\
\hline $\mathrm{S}^{\mathrm{xii}}-\mathrm{Ta}-\mathrm{Ta}^{\mathrm{xvi}}$ & $53.90(7)$ \\
\hline $\mathrm{S}^{\mathrm{iii}}-\mathrm{Ta}-\mathrm{Ta}^{\mathrm{xvi}}$ & $126.10(7)$ \\
\hline $\mathrm{S}-\mathrm{Ta}-\mathrm{Ta}^{\mathrm{xvi}}$ & $126.10(7)$ \\
\hline $\mathrm{S}^{\mathrm{xiii}}-\mathrm{Ta}-\mathrm{Ta}^{\mathrm{xvi}}$ & $53.90(7)$ \\
\hline $\mathrm{S}^{\mathrm{vii}}-\mathrm{Ta}-\mathrm{Ta}^{\mathrm{xvi}}$ & $53.90(7)$ \\
\hline $\mathrm{S}^{\mathrm{xiv}}-\mathrm{Ta}-\mathrm{Ta}^{\mathrm{xvi}}$ & $126.10(7)$ \\
\hline $\mathrm{Ta}^{\mathrm{xv}}-\mathrm{Ta}-\mathrm{Ta}^{\mathrm{xvi}}$ & 180.0 \\
\hline $\mathrm{Cu}^{\mathrm{xvi}}-\mathrm{Ta}-\mathrm{Ta}^{\mathrm{xvi}}$ & 0 \\
\hline $\mathrm{Cu}^{\mathrm{xv}}-\mathrm{Ta}-\mathrm{Ta}^{\mathrm{xvi}}$ & 180.0 \\
\hline $\mathrm{Ta}-\mathrm{S}-\mathrm{Cu}^{\mathrm{xv}}$ & 72.2 \\
\hline $\mathrm{Ta}-\mathrm{S}-\mathrm{Ta}^{\mathrm{xv}}$ & $72.19(13)$ \\
\hline $\mathrm{Cu}^{\mathrm{xv}}-\mathrm{S}-\mathrm{Ta}^{\mathrm{xv}}$ & 0 \\
\hline $\mathrm{Ta}-\mathrm{S}-\mathrm{Ba}$ & $89.64(7)$ \\
\hline $\mathrm{Cu}^{\mathrm{xv}}-\mathrm{S}-\mathrm{Ba}$ & $89.64(7)$ \\
\hline $\mathrm{Ta}^{\mathrm{xv}}-\mathrm{S}-\mathrm{Ba}$ & $89.64(7)$ \\
\hline $\mathrm{Ta}-\mathrm{S}-\mathrm{Ba}^{\mathrm{xvii}}$ & $89.64(7)$ \\
\hline $\mathrm{Cu}^{\mathrm{xv}}-\mathrm{S}-\mathrm{Ba}^{\mathrm{xvii}}$ & $89.64(7)$ \\
\hline $\mathrm{Ta}^{\mathrm{xv}}-\mathrm{S}-\mathrm{Ba}^{\mathrm{xvii}}$ & $89.64(7)$ \\
\hline $\mathrm{Ba}-\mathrm{S}-\mathrm{Ba}^{\mathrm{xvii}}$ & $179.11(16)$ \\
\hline $\mathrm{Ta}-\mathrm{S}-\mathrm{Ba}^{\mathrm{vi}}$ & $159.82(13)$ \\
\hline $\mathrm{Cu}^{\mathrm{xv}}-\mathrm{S}-\mathrm{Ba}^{\mathrm{vi}}$ & $87.629(7)$ \\
\hline
\end{tabular}




$\begin{array}{llll}\mathrm{S}^{\mathrm{iii}}-\mathrm{Ba}-\mathrm{S}^{\mathrm{xi}} & 89.75(5) & \mathrm{Ta} & 87.629(7) \\ \mathrm{S}^{\mathrm{iv}}-\mathrm{Ba}-\mathrm{S}^{\mathrm{xi}} & 89.75(5) & \mathrm{S}-\mathrm{Ba}^{\mathrm{vi}} & 90.25(5) \\ \mathrm{S}-\mathrm{Ba}-\mathrm{S}^{\mathrm{xi}} & 118.88(3) & \mathrm{Ba}^{\mathrm{xvii}}-\mathrm{S}-\mathrm{Ba}^{\mathrm{vi}} & 90.25(5) \\ \mathrm{S}^{\mathrm{v}}-\mathrm{Ba}-\mathrm{S}^{\mathrm{vi}} & 118.88(3) & \mathrm{Ta}-\mathrm{S}-\mathrm{Ba}^{\mathrm{ix}} & 87.629(7) \\ \mathrm{S}^{\mathrm{vi}}-\mathrm{Ba}-\mathrm{S}^{\mathrm{xi}} & 147.76(6) & \mathrm{Cu}^{\mathrm{xv}}-\mathrm{S}-\mathrm{Ba}^{\mathrm{ix}} & 159.82(13) \\ \mathrm{S}^{\mathrm{vii}}-\mathrm{Ba}-\mathrm{S}^{\mathrm{xi}} & 57.48(11) & \mathrm{Ta}^{\mathrm{xv}}-\mathrm{S}-\mathrm{Ba}^{\mathrm{ix}} & 159.82(13) \\ \mathrm{S}^{\mathrm{viii}}-\mathrm{Ba}-\mathrm{S}^{\mathrm{xi}} & 147.76(6) & \mathrm{Ba}-\mathrm{S}-\mathrm{Ba}^{a^{\mathrm{ix}}} & 90.25(5) \\ \mathrm{S}^{\mathrm{ix}}-\mathrm{Ba}-\mathrm{S}^{\mathrm{xi}} & 57.48(11) & \mathrm{Ba}^{\mathrm{xvii}}-\mathrm{S}-\mathrm{Ba}^{\mathrm{ix}} & 90.25(5) \\ \mathrm{S}^{\mathrm{x}}-\mathrm{Ba}-\mathrm{S}^{\mathrm{xi}} & 112.55(13) & \mathrm{Ba}^{\mathrm{vi}}-\mathrm{S}-\mathrm{Ba}^{\mathrm{ix}} & 112.55(13)\end{array}$

Symmetry codes: (i) $x+1, y, z$; (ii) $-y+1, x-y$, $z$; (iii) $-x+y,-x, z$; (iv) $-x+y+1,-x+1, z$; (v) $-y+1, x-y+1, z$; (vi) $-x+1,-y+1,-z+2$; (vii) $y,-x+y,-z+1$; (viii) $y,-x+y,-z+2$; (ix) $-x+1,-y+1,-z+1$; (x) $x-y+1, x,-z+2$; (xi) $x-y+1, x,-z+1$; (xii) $x-y, x,-z+1$; (xiii) $-x,-y,-z+1$; (xiv) $-y, x-y, z$; (xv) $-x,-y$, $z+1 / 2$; (xvi) $-x,-y, z-1 / 2$; (xvii) $x-1, y, z$. 